\title{
Deep-Learning-Based Intelligent Intervehicle Distance Control for 6G-Enabled Cooperative Autonomous Driving
}

\author{
Xiaosha Chen ${ }^{(\mathbb{1}}$, Supeng Leng ${ }^{(\mathbb{D}}$, Member, IEEE, Jianhua $\mathrm{He}^{(\mathbb{1})}$, and Longyu Zhou ${ }^{(\mathbb{C}}$
}

\begin{abstract}
Research on the sixth-generation cellular networks (6G) is gaining huge momentum to achieve ubiquitous wireless connectivity. Connected autonomous vehicles (CAVs) is a critical vertical application for $6 \mathrm{G}$, holding great potentials of improving road safety, road and energy efficiency. However, the stringent service requirements of $\mathrm{CAV}$ applications on reliability, latency, and high speed communications will present big challenges to $6 \mathrm{G}$ networks. New channel access algorithms and intelligent control schemes for connected vehicles are needed for $6 \mathrm{G}$-supported $\mathrm{CAV}$. In this article, we investigated 6G-supported cooperative driving, which is an advanced driving mode through information sharing and driving coordination. First, we quantify the delay upper bounds of $6 \mathrm{G}$ vehicle-to-vehicle $(\mathrm{V} 2 \mathrm{~V})$ communications with hybrid communication and channel access technologies. A deep learning neural network is developed and trained for the fast computation of the delay bounds in real-time operations. Then, an intelligent strategy is designed to control the intervehicle distance for cooperative autonomous driving. Furthermore, we propose a Markov chain-based algorithm to predict the parameters of the system states, and also a safe distance mapping method to enable smooth vehicular speed changes. The proposed algorithms are implemented in the AirSim autonomous driving platform. Simulation results show that the proposed algorithms are effective and robust with safe and stable cooperative autonomous driving, which greatly improve the road safety, capacity, and efficiency.
\end{abstract}

Index Terms-6G, connected autonomous driving, delay upper bound, distance control, stochastic network calculus (SNC).

\section{INTRODUCTION}

W ITH more than one million fatalities on the road globally and increasing global warming, there are urgent needs to reduce road accidents and energy consumption.

Manuscript received November 2, 2020; accepted December 23, 2020. Date of publication December 29, 2020; date of current version October 7, 2021 This work was supported in part by the National Key Research and Development Program of China under Grant 2018YFE0117500; in part by the National Natural Science Foundation of China under Grant 62071092; in part by the Science and Technology Program of Sichuan Province, China, under Grant 2019YFH0007; and in part by EU H2020 Project COSAFE under Grant MSCA-RISE-2018-824019. (Corresponding author: Supeng Leng.)

Xiaosha Chen and Supeng Leng are with the School of Information and Communication Engineering, University of Electronic Science and Technology of China (UESTC), Chengdu 611731, China and the Shenzhen Institute for Advanced Study, UESTC (e-mail: spleng@uestc.edu.cn).

Jianhua $\mathrm{He}$ is with the School of Computer Science and Electronic Engineering, University of Essex, Colchester CO4 3SQ, U.K.

Longyu Zhou is with the School of Information and Communication Engineering, University of Electronic Science and Technology of China, Chengdu 611731, China.

Digital Object Identifier 10.1109/JIOT.2020.3048050
Since more than $90 \%$ road accidents were caused by human errors [1], autonomous driving, which is boosted by advanced sensors and artificial intelligence (AI) technologies, is widely accepted as one of the most promising technologies to enhance road safety. However, due to the inherent perception limitations of sensors and the complicated driving conditions, autonomous driving brings its own road safety problems, there is still a long way to go for full autonomous driving. More recently, connected autonomous vehicles (CAVs) with cooperative sensing and driving were proposed as a new paradigm for future transports [2]-[6].

Vehicle-to-vehicle (V2V) communication plays a critical role for the cooperative driving. 6G-enabled CAVs are required to have ultrashort communication latency, ultrahigh reliability, extremely broad bandwidth, intelligent network traffic control, road perception, and smart decision-making functions to support new on-road applications. Meanwhile, research on $6 \mathrm{G}$ is gaining huge momentum to achieve up to $1 \mathrm{~Tb}$ per second and ubiquitous wireless connectivity. The candidate-enabling technologies include Terahertz, space-terrestrial networking, reconfigurable intelligent surface, and AI [7], [8]. However, new channel access algorithms and intelligent control schemes for connected vehicles are needed to meet the stringent communication requirements of CAV applications.

As safety is ultimately important for on-road driving applications, it is also necessary to fully model the $\mathrm{V} 2 \mathrm{~V}$ communication performance and explore its impact on the safety of the CAV applications, especially under the poor network conditions. To the best of our knowledge, there is no reported work on the modeling and quantifying the performance of $\mathrm{V} 2 \mathrm{~V}$ communications within a heterogeneous vehicular network with multiple communication technologies.

In view of the above research challenges, we are motivated to investigate the communication performance of $6 \mathrm{G}$ V2V networks with hybrid communication and channel access schemes, and apply the findings to the intelligent control of cooperative driving. Cooperative driving is taken as a representative CAV driving mode, which is enabled through information sharing and driving coordination among vehicles. First, we analytically model the communication delay upper bounds of the V2V networks by the stochastic network calculus (SNC), which is a popular mathematical tool to analyze the performance low bound of wireless communication systems. While the analytic model is effective and helpful, the high computational time prevents its use in real-time network 
operation. To address this problem, a deep learning neural network is developed and trained for the fast prediction of the delay bounds in real-time operations. The analytical results obtained from the SNC are used to create training samples for the deep learning neural network. With the newly available V2V communication performance bounds, we propose an intelligent strategy for the control of intervehicle distance for cooperative autonomous driving. Furthermore, we propose a Markov chain-based algorithm to predict the parameter and a safe distance mapping method, which are utilized to enable smooth vehicular speed changes [9].

Our contributions are summarized as follows.

1) We propose an SNC-based theoretical tool set to derive the communication delay upper bounds for a V2V network with hybrid communication technologies. Furthermore, a new analytical paradigm combining with the deep neural network and SNC theory is proposed for the low bound calculation of the real-time performance in a CAV environment.

2) With the communication latency bounds, we propose an intelligent intervehicle distance control strategy for CAV. With the aid of the pretrained deep neural network and our proposed online prediction method, each vehicle can determine its speed individually based on the states of other vehicles. Parameter prediction and distances mapping algorithms are designed to enable smooth speed changes, which can improve the efficiency of road traffic and energy consumption.

3) We implement a comprehensive simulation platform that integrates AirSim-based cooperative autonomous driving, Mathematica-based SNC computing, communication, and machine learning simulation. Extensive simulation experiments demonstrate that the proposed intelligent strategy works excellently in CAV scenarios. It is effective and robust in terms of safety and stability with smooth speed changes.

The remainder of this article is organized as follows. Section II introduces the related work of connected vehicles and autonomous driving. Section III presents the system model and assumptions. Section IV shows the analysis of the proposed 6G-enabled cellular vehicular network. We present our proposed vehicular control algorithm in Section V. Numerical simulation results and discussions are presented in Section VI. Finally, Section VII concludes this article.

\section{RELATED WORK}

Road accidents and traffic congestion have been global challenges nowadays. Equipped with advanced sensors and deep learning technologies, the driving of modern vehicles is becoming much autonomous and safe. However, due to the limitations of the sensors (such as limited sensing ranges and poor performance of cameras and radars in adverse weather conditions), the safety of these vehicles is still far from being satisfactory. On the other hand, connected vehicles are opening space for cooperative driving, which can be an excellent complement to provide a critical virtual sensor for autonomous vehicles [10]-[13].
The deep reinforcement learning technology has been widely adopted in on-road scenarios. Chen et al. [14] proposed a smart task offloading scheme for mobile devices with dense deployment of RSUs. Using deep reinforcement learning algorithms, mobile devices can decide whether their tasks will be offloaded, and how to optimize the route from devices to MEC servers. In [15], the bandwidth allocation problem of a vehicular network is modeled as a discrete-time singleagent Markov decision process, which is solved by a deep learning-based approach. Chen et al. [16] proposed a deep reinforcement learning-based framework to overcome dynamic blockage in the mmWave-based V2X network and improve the reliability of the mmWave communications. Tang et al. [17] discussed the future direction of the V2X technologies in $6 \mathrm{G}$ networks. The survey concluded that machine learning techniques will be applied in the communication, networking, and security approaches in future $6 \mathrm{G}$ vehicular networks. AIenabled algorithms are expected to be widely deployed in $6 \mathrm{G}$ vehicular communication technologies. Zhou et al. [18] discussed future big data-enabled V2X technologies. With the big data and cloud-driven framework, smart algorithms can be easily implemented in on-road scenarios.

As a promising technology for improving road efficiency and safety, 6G-based V2V communications were studied by a few works. Yuan et al. evaluated the efficiency of $6 \mathrm{G}$ fullduplex V2V channels in [19]. Tang et al. provided a survey on the application of machine learning for $6 \mathrm{G}$ vehicular network. However, research on $6 \mathrm{G}$ technologies is still in an early stage. There is no report on the new design of channel access for the $6 \mathrm{G} \mathrm{V} 2 \mathrm{~V}$ networks with hybrid communication technologies.

Some previous works studied the delay upper bound for different types of vehicular networks. Xie and Jiang [20] obtained the stochastic delay upper bound for the IEEE 802.11 DCF network, which formed the basis of many following works. The work [21] studied the end-to-end delay upper bound for the V2X network. However, they only consider the DSRC for the V2V network, without a consideration of the mmWave communication part. Yang et al. analyzed the multihop mmWave networks with full-duplex buffered relays in [22], where the moment generating function (MGF)-based upper bound for the service curve of the mmWave network was obtained. However, the analysis of the V2V delay upper bound in the previous works did not consider the hybrid access $\mathrm{V} 2 \mathrm{~V}$ network, which is consisted of different types of $\mathrm{V} 2 \mathrm{~V}$ communications links.

Zegers et al. proposed an intervehicle distance control strategy based on the sensor data in [25] by extending the work in [26]. Nevertheless, the real-time sensor data-based control strategy fluctuates in a relatively wide range, which reduces the road and energy efficiency.

In summary, the performance analysis and traffic control have been investigated separately in the previous research work. Besides, the existing network calculus-based analytical methods for the delay upper bound have very high computational complexity. Therefore, it is difficult to apply these methods in online algorithms. The machine learning technology is a promising way to implement real-time SNC-based algorithms, which is one of the main ideas of this article. 
TABLE I

CRITICAL PARAmeters

\begin{tabular}{|c|c|}
\hline Parameter & Description \\
\hline$p_{c}$ & Saturated packet transmission collision probability \\
\hline $\bar{t}_{\text {serv }}$ & Mean service time with exponential back-off algorithm \\
\hline$n$ & Number of vehicles \\
\hline$\mu_{k}$ & Average size of contention window at back-off stage $k$ \\
\hline$t_{s}$ & Average time for successful transmission \\
\hline$t_{C}$ & Average time for collision \\
\hline$L$ & Queue length \\
\hline$l$ & Distance between adjacent vehicles \\
\hline$M$ & Maximum back-off times \\
\hline $\mathcal{B}$ & Total back-off content window size \\
\hline$d(t)$ & Virtual delay for corresponding arrival process \\
\hline$h(\alpha, \beta)$ & Maximum horizontal distance between function $\alpha$ and $\beta$ \\
\hline$\lambda$ & Packet arrival rate \\
\hline$A_{a}, A_{d}$ & Accelerate and decelerate speed in vehicle control scheme \\
\hline$k_{1}, k_{2}$ & Distance mapping factor in safe distance mapping algorithm \\
\hline$v_{i}$ & Speed of vehicle $i$ \\
\hline$S_{i, i+1}$ & Safe distance between vehicle $i$ and vehicle $i+1$ \\
\hline
\end{tabular}

\section{SySTEM MODEL}

In this section, we introduce the system model and the basic assumptions. As base stations may not be always available in all scenarios, we consider the worst cases of the vehicle networks for cooperative driving, where vehicles communicate through distributed V2V communications. Some critical symbols are summarized in Table I.

In the distributed $\mathrm{V} 2 \mathrm{~V}$ communication networks, vehicles compete to access the broadcast channels with conventional for data transmission in a random manner, as specified in the DSRC and 3GPP LTE-V2V technologies. In this article, we will take exponential back-off protocols as the random access protocols for communication performance modeling and design of intervehicle distance control strategies. It is noted that the proposed theory model in the paper is applicable to modern random access protocols for $6 \mathrm{G}$ V2 $\mathrm{V}$ networks as well. Furthermore, to improve the communication reliability and data rate, we assume that vehicles are also equipped with $6 \mathrm{G}$ radio access device, such as the visible-light communication unit, on top of the traditional $5 \mathrm{G}$ radio access device.

For the sake of analytical model tractability, hidden node and exposed node problems are not investigated as done in the most previous analytical works. For the infrastructure-free $\mathrm{V} 2 \mathrm{~V}$ communication at relative low-radio frequency, all vehicles follow the random channel access for data transmissions. They are assumed to be within the communication range of each other. While for the $6 \mathrm{G}$ V2V communication at highfrequency bands (such as mmWave and Terahertz bands), due to the line-of-sight communication limitation, we assume that the vehicles can only communicate to the vehicles immediately next to them and within the communication range. Without loss of generality, we will take mmWave communication as an example for $6 \mathrm{G} \mathrm{V} 2 \mathrm{~V}$ communication at high-frequency bands. Random channel access is not applied for $6 \mathrm{G} \mathrm{mmWave}$ communications as there is no contention exists with other vehicles for communication with beamforming technologies. But the random channel access-based V2V communications at the low-frequency bands will be used for the direct communications between vehicles. To differentiate the random

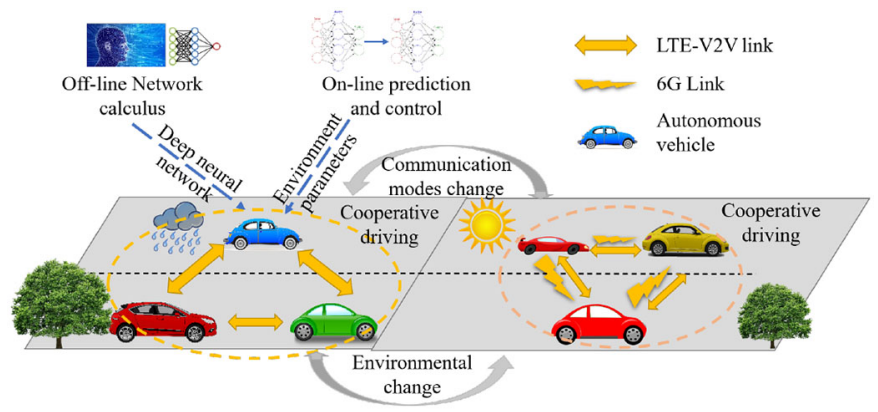

Fig. 1. Scenario of 6G-supported cooperative connected vehicles.

channel access communications at low frequency and the direct communication at high-frequency bands, we call these communications as cellular $\mathrm{V} 2 \mathrm{~V}$ and $6 \mathrm{G}$ communications, respectively. The V2V networks with both low-frequency band and 6G higher frequency band are called hybrid networks. The protocols and topology of the hybrid network will be presented along with the explanation of some essential assumptions.

Fig. 1 shows an example 6G-supported vehicular network under different weather conditions. In the good weather conditions, both cellular V2V and 6G communication channels can be established for short safety messages and large messages containing sensor data, separately. However, if the environment is not feasible to establish the $6 \mathrm{G}$ channels, the cellular $\mathrm{V} 2 \mathrm{~V}$ can also maintain the basic functions of the cooperative autonomous driving. According to the characteristics of cellular $\mathrm{V} 2 \mathrm{~V}$ and $6 \mathrm{G}$ communications, it is reasonable to transmit short packets for wide range through cellular V2V communications and large sensing data packets for close neighbors via $6 \mathrm{G}$ communications. If the $6 \mathrm{G}$ connectivity is not available, cellular $\mathrm{V} 2 \mathrm{~V}$ communication is needed to transfer part of the sensor data. For the hybrid vehicular network, each vehicle has a message queue for cellular V2V communication and $6 \mathrm{G}$ communication separately. The vehicles have identical capacity of queues for the same communication type. The queue length of cellular V2V communication is denoted by $L$. Vehicles can establish $6 \mathrm{G}$ communication links only if the distance between them is shorter than a required threshold, such as the mmWave link in [27]. To simplify the performance analysis, we assume that the topology of the $6 \mathrm{G}$ communication network is a connected graph. The $6 \mathrm{G}$ communication is assumed as a fluid-flow, discrete-time queuing system with an infinite buffer. Moreover, the longest flow in the $6 \mathrm{G}$ network is a flow with a start node $S, n-2$ full-duplex relays, and a destination node $D$, where $n$ is the number of vehicles.

\section{A. Cellular V2V Communication}

For the cellular V2V communication, the system time is slotted. The communication follows the exponential back-off mechanism like IEEE 802.11p standard. In the back-off stage, we assume each communication process at a vehicle is in one of $M+1$ states, denoted by $B_{0}, B_{1}, \ldots, B_{M}$, respectively. Each communication process also maintains a back-off counter and a content window. In the initial state $B_{0}$, the content window size is set to $\mathrm{CW}_{0}=\mathrm{CW}_{\text {min }}=W$. The back-off counter is uniformly and randomly picked in the range $\left[0, \mathrm{CW}_{0}\right]$. The 
back-off counter decreases by one if the channel is idle and will be frozen when the channel is busy. A vehicle tries to transmit the packet if its back-off counter equals 0 . If the transmit packet collides with other packet(s), the vehicle will transform to the next state and reinitialize the back-off counter in the range $\left[0, \mathrm{CW}_{i}\right]$, where $i$ is the current state index. If a conflict appears in the last state, the back-off process will start again from the initial state. The value of $\mathrm{CW}_{i}$ can be obtained by $\mathrm{CW}_{i}=\min \left\{2^{i} W, 2^{m} W\right\}$, where $0<m \leq M$ is a parameter of the protocol to control the maximum content window size.

Note that for data broadcasting, messages are usually not retransmitted due to the difficulty of sending back ACK or NACK packets. For many safety applications, periodic broadcast messages will need to carry the latest vehicle status information (such as location, speed, driving direction etc.), retransmitted messages with excessive delay could be outdated and not useful for the safety applications. However, the back-off mechanism is still applied to the transmission of all packets for their first transmission attempt, which is important to control channel congestion, especially for distributed $\mathrm{V} 2 \mathrm{~V}$ communications.

\section{B. $6 G$ Communication}

As a typical example of $6 \mathrm{G}$ communication links, we consider the mmWave link in this section. As other types of 6G communication channels mainly differ on the channel model, the analysis will be similar. We assume a homogeneous mmWave communication environment, where all the mmWave links follow the same fading distribution. Given the distance between the nodes, a general fading factor $g$ in $\mathrm{dB}$ is given by [28], [29]

$$
g[\mathrm{~dB}]=-\left(\alpha+10 \beta \log _{10}(l)+\xi\right)
$$

where $\alpha$ and $\beta$ are the least square fits of the floating intercept and slope of the best fit, and $\xi \sim \mathcal{N}\left(0, v^{2}\right)$, a log-normal shadowing effect with variance $v^{2}$. The parameters $\alpha$ and $\beta$ are dependent on the communication environment.

Furthermore, as the mmWave channels are full-duplex, selfinterference needs to be considered. This article adopts a widely used method to model the self-interference, which uses a coefficient $0 \leq \mu \leq 1$ to characterize the coupling between the transmitter and receiver. The interference plus noise ratio (SINR) can be denoted by $\gamma$, which is computed by $\gamma=\kappa \cdot \omega \cdot g$, where

$$
\omega= \begin{cases}\frac{\mathrm{SNR}}{1+\mu \mathrm{SNR}}, & i \in\{1,2, \ldots, n-1\} \\ \mathrm{SNR}, & i=n\end{cases}
$$

and SNR $=(P) /\left(N_{0}\right)$ denotes the transmitted signal-tonoise ratio (SNR), where $P$ and $N_{0}$ are the transmitted power and background noise, respectively.

\section{COMmunication Delay Upper Bounds}

To determine the intervehicle safe distance, the communication delay upper bound needs to be computed. In this section, we will analyze the stochastic delay upper bound of hybrid V2V networks as introduced in Section III. We first show the performance analysis of the $\mathrm{V} 2 \mathrm{~V}$ network with only cellular
V2V communication or $6 \mathrm{G}$ mmWave communication, respectively. Then, we present the analysis of the proposed hybrid network with SNC theory. All queues studied in this article are first-in-first-out (FIFO) queues.

\section{A. Performance of Cellular V2V Communications}

To analyze the performance of the $\mathrm{V} 2 \mathrm{~V}$ network with cellular $\mathrm{V} 2 \mathrm{~V}$ communication, the collision probability will be analyzed first. According to [30], we have the following formula for the collision probability, which is denoted by $p_{c}$ :

$$
\begin{aligned}
& p_{c}:=1-e^{-(n-1) p_{a}} \\
& p_{a}:=\frac{\sum_{k=0}^{M} p_{c}^{k}}{\sum_{k=0}^{M} \mu_{k} p_{c}^{k}}
\end{aligned}
$$

where $p_{c}$ and $p_{a}$ are the probabilities that a collision happens in a time slot and a vehicle tries to transmit in a time slot, respectively. $\mu_{k}$ is the average size of the content window size of back-off stage $k$. The collision probability $p_{c}$ can be obtained by solving the nonlinear equation system in (3), which is very fast by the numerical method.

We can calculate the mean service time for the random access channel by [21]

$$
\bar{t}_{\mathrm{serv}}=\sum_{j=0}^{M} p_{c}^{j} \bar{t}_{j}
$$

where $\bar{t}_{j}$ is the mean time that a node stays at back-off stage $j$, which can be calculated by

$$
\bar{t}_{j}=\mu_{j} t_{B}+t_{T X}
$$

where $t_{B}$ is the average length of a back-off slot, and $t_{T X}$ is the average length of a transmission slot. We can obtain $t_{B}$ by

$$
t_{B}=\left(1-p_{a}\right)^{n}+n \cdot p_{a}\left(1-p_{a}\right)^{n-1} t_{T X}+p_{c} t_{C}
$$

where $t_{C}$ is the length of the collision back-off slot.

For the FIFO queue, the average waiting time can be obtained by the basic queue theory. We assume that the queue is a G/M/1 queue and according to the Pollaczek-Khinchin formula, the mean waiting time is

$$
t_{q}=\rho+\frac{\rho^{2}+\lambda^{2} \operatorname{Var}(S)}{2(1-\rho)}
$$

where $\lambda$ is the arrival rate for the Poisson process, $\rho=\lambda \bar{t}_{\text {serv }}$ is the utilization, and $\operatorname{Var}(S)$ is the variance of the service time distribution $S$.

In terms of the concatenation property of SNC, and according to the work in [20], we can express the service curve of a node in cellular $\mathrm{V} 2 \mathrm{~V}$ network when $0 \leq y<1-q$ with

$$
\begin{aligned}
\beta(t) & =\bar{t}_{\mathrm{serv}} \lambda t \otimes t_{q} \lambda t \\
g_{t}(x) & =\left\{\left(\frac{q}{y}\right)^{y}\left(\frac{1-q}{1-y}\right)^{1-y}\right\}^{L}
\end{aligned}
$$

where

$$
\begin{aligned}
& q=\frac{\bar{t}_{\mathrm{serv}}+t_{q}-t_{s}}{M t_{C}+L \bar{t}_{\mathrm{serv}}+\mathcal{B} t_{s}} \\
& y=\frac{x-L t_{s}}{L\left(M t_{C}+L \bar{t}_{\mathrm{serv}}+\mathcal{B} t_{s}\right)}
\end{aligned}
$$


where $\mathcal{B}=\sum_{k=0}^{M}\left(C W_{k}-1\right), t_{s}$ is the average time that the channel is busy due to the successful transmission. $\otimes$ is the min-plus convolution in network calculus, which is defined by $(\alpha \otimes \beta)(t)=\inf _{0 \leq \tau \leq t}\{\alpha(\tau)+\beta(t-\tau)\}$. Based on the SNC theory, the service process $S(t)$ of a node can be expressed by the stochastic service curve $\beta(t)$ bounded by $g_{t}(x)$, which is written as $S \sim_{\mathrm{sc}}\left\langle g_{t}, \beta\right\rangle$, and is defined as $P\left\{\sup _{0 \leq s \leq t}[A \otimes\right.$ $\left.\left.\beta(s)-A^{*}(s)\right]>x\right\} \leq g_{t}(x)$, where $A(t)$ and $A^{*}(t)$ are the arrival and departure processes, respectively.

\section{B. Performance of $6 G$ Communication Networks}

To simplify the analysis of the delay with $6 \mathrm{G}$ mmWave communication, which is assumed to be mmWave links as an typical example, we assume that all of the background noises for the links have the same power, which is called the homogeneous mmWave network. As for the arrival process of the mmWave network, we assume that it is a $(\rho, \theta)$-upper bounded stochastic arrival process, which is defined as

$$
M_{A}(\theta, s, t) \leq e^{\theta(\rho(\theta)(t-s)+\sigma(\theta))} \triangleq e^{\theta \sigma(\theta)}\left(p_{a}(\theta)\right)^{t-s}
$$

where $M_{A}(\theta, s, t)$ is the moment of the arrival process in time period $[s, t)$ with respect to $\theta$ and $p_{a}(\theta)=e^{\theta \rho(\theta)}$.

According to the work in [22], the MGF of the service process in the $n$-hop mmWave network has an upper bound, which is

$$
\mathrm{M}(\theta, s, t) \leq \frac{e^{\theta \sigma(\theta)}}{p_{a}^{s-t}(\theta)} \cdot \mathcal{G}_{\tau, n-1}\left(p_{a}(\theta) \hat{q}(-\theta)\right)
$$

for any $\theta>0$, where $\tau \triangleq \max (s-t, 0)$ and $p_{a}(\theta) \hat{q}(-\theta)<1$. Moreover, $\hat{q}(-\theta)$ is defined as the exception of $(1+\xi)^{-\theta}$, which can be calculated by

$$
\begin{aligned}
\hat{q}(-\theta) & =\mathbb{E}\left[(1+\xi)^{-\theta}\right] \\
& \approx \min _{u \geq 0}\left\{\left(1+\delta N_{\delta}(u)\right)^{-\theta}+\sum_{i=1}^{N_{\delta}(u)} a_{\theta, \delta}(i) F_{\xi}(i \delta)\right\}
\end{aligned}
$$

where $F_{\xi}(x)$ is the probability density function (PDF) of random variable $\xi, N_{\delta}(u)=\lfloor(u) /(\delta)\rfloor$ and $a_{\theta, \delta}(i)=(1+(i-$ 1) $\delta)^{-\theta}-(1+i \delta)^{-\theta}$, and $\delta$ is a parameter, less $\delta$ means higher accuracy.

Moreover, in (12), $\mathcal{G}_{\tau, n}$ is defined as

$$
\mathcal{G}_{\tau, n}(x) \triangleq \min \left\{\mathcal{G}_{1}(x), \mathcal{G}_{2}(x)\right\}
$$

where

$$
\begin{aligned}
& \mathcal{G}_{1}(x)=\frac{\min \left(1, x^{\tau}\left(\begin{array}{c}
n+\tau \\
n
\end{array}\right)\right)}{(1-x)^{n+1}} \\
& \mathcal{G}_{2}(x)=\frac{1}{(1-x)^{n+1}}-\left(\begin{array}{l}
n+\tau \\
n+1
\end{array}\right) x^{\tau-1} .
\end{aligned}
$$

After we obtain the MGF bound, we can get a probability upper bound by the Chernoff Bound, which is

$$
P\{X \leq x\} \leq e^{-\theta x} E e^{\theta X}=e^{\theta x} M_{X}(-\theta) .
$$

Consequently, the service amount of the mmWave network can be written as

$$
P\{X \leq x\} \leq \frac{e^{\theta(\sigma(\theta)+x)}}{p_{a}^{s-t}(\theta)} \cdot \mathcal{G}_{\tau, n-1}\left(p_{a}(\theta) \hat{q}(-\theta)\right) .
$$

Replacing $x$ with $\beta(t-s)-x$ in (18), we can get

$$
\begin{aligned}
P\{X & \leq \beta(t-s)-x\} \\
& \leq \frac{e^{\theta(\sigma(\theta)+\beta(t-s)-x)}}{p_{a}^{s-t}(\theta)} \cdot \mathcal{G}_{\tau, n-1}\left(p_{a}(\theta) \hat{q}(-\theta)\right) .
\end{aligned}
$$

Let $\beta(t-s)=(1 / \theta) \ln p_{a}^{s-t}(\theta)=(t-s) \rho$, we can get the service curve for the mmWave network

$$
\begin{aligned}
P\{X & \leq \rho(t-s)-x\} \\
& \leq e^{\theta(\sigma(\theta)-x)} \cdot \mathcal{G}_{\tau, n-1}\left(p_{a}(\theta) \hat{q}(-\theta)\right) .
\end{aligned}
$$

From (20) and [31], we can find that the mmWave network provides a weak stochastic service curve for the flows.

\section{Delay Upper Bounds}

After obtaining the service curve, we can get the delay upper bound. According to [31, Th. 4.4], a virtual backlogcentric service curve is also a traffic-centric service curve. Consequently, we can directly consider (8) and (9) as the traffic-centric service curve. For the arrival curve $\alpha(t)$ with bounding function $f(x)$ and the service curve $\beta(t)$ with bounding function $g(x)$, the virtual delay $d(t)$, which is defined as

$$
d(t) \triangleq \inf \left\{\tau: A(t) \leq A^{*}(t+\tau)\right\}
$$

can be calculated by

$$
P\{d(t)>h(\alpha(t)+x, \beta(t))\} \leq(f \otimes g)(x) .
$$

For the parallel service curves 1 and 2, which are given by

$$
\begin{aligned}
& P\left\{X \leq \beta_{1}(t-s)-x\right\} \leq f_{1}(x) \\
& P\left\{X \leq \beta_{2}(t-s)-x\right\} \leq f_{2}(x)
\end{aligned}
$$

where $f_{1}(x)$ and $f_{2}(x)$ are the two bounding functions of the parallel service curve, respectively. The total service curve can be obtained by

$$
P\left\{X \leq \beta_{1}(t-s)+\beta_{2}(t-s)-2 x\right\} \leq f_{1} * f_{2}(x)
$$

where $*$ is the Stieltjes convolution, which is defined as

$$
(a * b)(x) \triangleq \int_{-\infty}^{\infty} a(x-y) d b(y) .
$$

Replacing $2 x$ with $y$ in (24), we can obtain

$$
P\left\{X \leq \beta_{1}(t-s)+\beta_{2}(t-s)-y\right\} \leq f_{1} * f_{2}\left(\frac{y}{2}\right) .
$$

From (8), (9), (20), and (26), we can get the stochastic service curve bound for the hybrid $\mathrm{V} 2 \mathrm{~V}$ network, which is

$$
\begin{aligned}
P\{X \leq & \left.\bar{t}_{\mathrm{scrv}} \lambda t \otimes t_{q} \lambda t+\rho(t-s)-x\right\} \\
\leq & -\frac{\theta}{2} \mathcal{G}_{\tau, n-1}\left(p_{a}(\theta) \hat{q}(-\theta)\right) \\
& \times \int_{-\infty}^{\infty}\left\{\left(\frac{q}{y^{\prime}}\right)^{y^{\prime}}\left(\frac{1-q}{1-y^{\prime}}\right)^{1-y^{\prime}}\right\}^{L} e^{\theta(\sigma(\theta)-x / 2)} \mathrm{d} x
\end{aligned}
$$

where

$$
y^{\prime}=\frac{x / 2-L t_{s}}{L\left(M t_{C}+L \bar{t}_{\mathrm{serv}}+\mathcal{B} t_{s}\right)} \text {. }
$$


With (22) and (27), and according to the aggregation property of the arrival process in [31], we can obtain the stochastic delay upper bound. Moreover, with the same method, the delays of the pure cellular V2V and pure $6 \mathrm{G}$ vehicular network can also be obtained.

\section{Distance Control Strategy for Cooperative CONNECTED DRIVING}

Based on the delay upper bound, we can develop an intelligent intervehicle distance control strategy for connected vehicles on one lane as shown in Fig. 1. The proposed strategy needs to satisfy three requirements on safety, traffic efficiency, and energy efficiency. Because of the high complexity of the delay computation, we use first devise a machine learningbased approach to make the algorithm suitable for real-time operation. We will split the control strategy into two parts: 1) the calculation of safe distance and 2) decision making.

\section{A. Parameter Prediction}

To improve the safety level of the traffic and improve the on-road experiment, the communication performance of the vehicular network needs to be predicted. In this section, we will present a simple but efficient and accurate neural networkbased prediction algorithm.

Let $X$ be a discrete random process, and there are $X_{n}$ states that the process $X$ can be. Moreover, the time space is also discrete. At time $t, X$ generates a value $x_{t}$, and the value of $x_{t}$ depends on the previous $n$ values, in other words, $X$ is an $n$-order Markov process, which means

$$
\begin{aligned}
& P\left(x_{t} \mid x_{t-1}, x_{t-2}, \ldots, x_{0}\right) \\
& \quad=P\left(x_{t} \mid x_{t-1}, x_{t-2}, \ldots, x_{t-n}\right) .
\end{aligned}
$$

If the values of a parameter are continuous, such as the distance of the vehicles, we can discretize the values by dividing the real number into different levels. To learn the transition probability in (29), the training data need to be gathered first. With enough training data, the transition frequency can be calculated by

$$
\hat{P}\left(\hat{x}_{t} \mid \hat{x}_{t-1}, \ldots, \hat{x}_{t-n}\right)=\frac{C\left(\hat{x}, \hat{x}_{t-1}, \ldots, \hat{x}_{t-n}\right)}{C\left(\hat{x}_{t-1}, \ldots, \hat{x}_{t-2}\right)}
$$

where $\hat{P}\left(\hat{x}_{t} \mid \hat{x}_{t-1}, \ldots, \hat{x}_{t-n}\right)$ means the frequency of $\hat{x}_{t}$ when the previous $n$ values are $\hat{x}_{t-1}, \ldots, \hat{x}_{t-n}$, respectively. $C\left(x_{1}, \ldots, x_{n}\right)$ is the number of sequence $x_{1}, \ldots, x_{n}$ in the gathered data.

Learning the transition probability is essentially a data fitting problem. As the number of possible combinations of the $n$ values is too high in most cases, we apply a deep neural network method to predict the transition probability. The inputs of the neural network are $n$ previous values, respectively, and the outputs of the neural network are the probability of $X_{n}$ states. However, if we directly use the results of (30) to train the neural network, the deep neural network can be easily overfitted as the frequency is not accurate when the data size is small. We formulate a confidence factor to avoid this problem. A confidence factor of sequence $x_{1}, \ldots, x_{n}$ is defined as

$$
F\left(x_{1}, \ldots, x_{n}\right)=1-e^{\zeta \cdot C\left(x_{1}, \ldots, x_{n}\right)}
$$

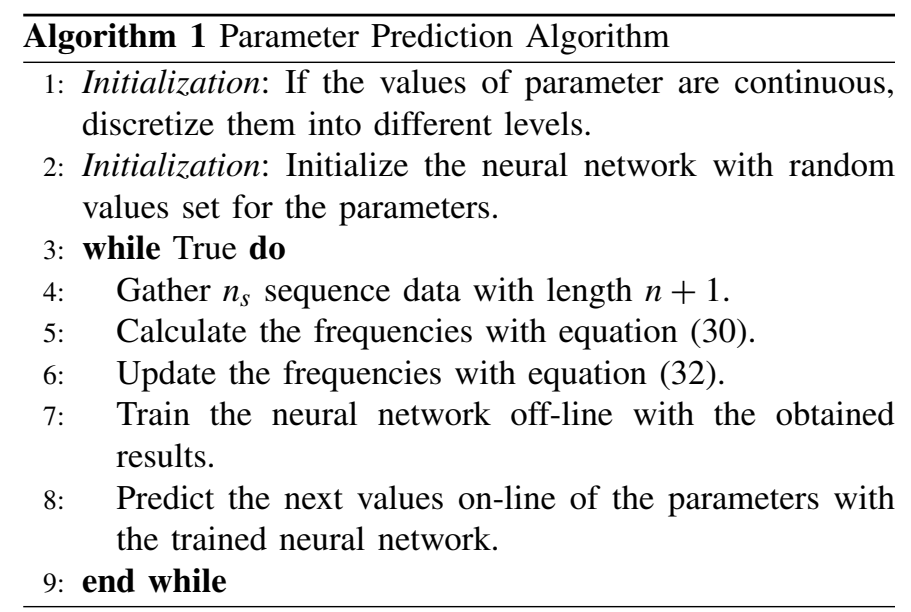

where $\zeta$ is a negative real number that can improve the accuracy. According to (31), the frequencies of sequence $x_{1}, \ldots, x_{n}$ need to be updated according to the following equation:

$$
\hat{P}_{u}\left(\hat{x} \mid x_{n}, \ldots, x_{1}\right)= \begin{cases}\hat{P}-\frac{F\left(x_{1}, \ldots, x_{n}\right)}{Z\left(x_{1}, \ldots, x_{n}\right)}, & \hat{P}>0 \\ \frac{F\left(x_{1}, \ldots, x_{n}\right)}{Z\left(x_{1}, \ldots, x_{n}\right)}, & \hat{P}=0\end{cases}
$$

where $\hat{P}=\hat{P}\left(\hat{x} \mid x_{n}, \ldots, x_{1}\right)$ and $Z\left(x_{1}, \ldots, x_{n}\right)$ is the number of states that the frequency equals 0 when the previous values are $x_{1}, \ldots, x_{n}$ in the state space.

In this article, we use a deep neural network structure with four layers, all the nodes in the neural networks are ramp nodes. The whole process of the proposed prediction algorithm is presented in Algorithm 1.

\section{B. Distance and Speed Control}

The core task of the vehicular distance control strategy is to decide the distance between the vehicles and the speed of each vehicle. In this section, we will present how to determine the safe distance according to the results in Section IV and how to avoid the high computation complexity of the network calculus by using the deep neural network.

The safe distance means if the preceding vehicle starts to brake at its highest acceleration, then after receiving the control message, the current vehicle behind the preceding vehicle starts to brake, too. Any collisions need to be avoided in this process. According to this description, we can formulate the safe distance as following equation:

$$
S_{i, i+1}=\frac{v_{i}^{2}}{2 a_{i}}+D_{i, i+1} \cdot v_{i}-\frac{v_{i+1}^{2}}{2 a_{i+1}}
$$

where $v_{i}$ and $v_{i+1}$ are the speed of the current vehicle and preceding vehicle, respectively. $a_{i}$ and $a_{i+1}$ are the maximum acceleration of the current and preceding vehicles, respectively. $D_{i, i+1}$ is the delay upper bound calculated by the network calculus.

However, different from the wired link, the wireless communication is a stochastic process, it is not possible to provide an exactly delay upper bound. As we can see in Section IV, the delay upper bound of the vehicular network is a probability distribution. Consequently, according to the dangerous level of different roads, the time-out probability upper bound 
$p$ needs to be provided. For example, on a road that traffic accident happens frequently, we need the time-out probability cannot be higher than 0.01 , which means $p=0.01$. On the other hand, if the road is safer, $p$ can be lower, such as 0.1 .

Nevertheless, calculating the delay upper bound given $p$ according to the network calculus needs dramatic computation resources and will lead to high computation delay. The safe distance decision process needs to be finished at a ultrafast speed to guarantee the road safety and make the algorithm online. Our solution calculates the delays of different parameters and time-out probabilities off-line, then train a network with given data. The input of the neural network is the communication parameters and the probability, the output is the delay upper bound. Because the calculation is environment independent, this calculation even can be finished at the cloud and the results can be downloaded by vehicles before they run on roads.

If we adjust the distance between vehicles according to the safe distance, because of the high dynamics of the environment, the distance will change at each time, which will make the vehicles change their speeds frequently. This will cause vehicular energy wasting and reduce the on-road experience. Furthermore, the frequent acceleration and deceleration will cause phantom traffic jams, which will heavily reduce the road traffic efficiency. To reduce the speed change, we adopt two methods: 1) parameter prediction and 2) safe distance mapping.

In the safe distance process, we consider not only the current safe distance, but also the longest safe distance in the next $T_{n}$ time by parameter prediction. Vehicles will predict the network parameters individually with Algorithm 1, then calculate the delay upper bounds and the safe distances with the trained neural network by (33), and adjust the speed according to the worst case.

To further reduce the speed changing, we apply the safe distance mapping algorithm. If the current distance $d_{i, i+1}$ between a vehicle and its preceding vehicle satisfy $k_{1} \cdot S_{i, i+1}<d_{i, i+1}<$ $k_{2} \cdot S_{i, i+1}$, where $k_{2}>f_{k}>1$, the acceleration of this vehicle should be 0 . The acceleration-distance relationship is illustrated in Fig. 2 and can be expressed by the following equation:

$$
a_{i}= \begin{cases}A_{a}, & d_{i, i+1}>k_{2} \cdot S_{i, i+1} \\ 0, & k_{1} \cdot S_{i, i+1}<d_{i, i+1}<k_{2} \cdot S_{i, i+1} \\ A_{d}, & d_{i, i+1}<k_{1} \cdot S_{i, i+1}\end{cases}
$$

where $A_{a}$ is a positive number and $A_{d}$ is a negative number, which can be chosen according to the traffic. Combining the parameter prediction, distance mapping and neural network calculate delay upper bound, the whole intelligent distance control strategy is given by Algorithm 2. The flowchart of the whole process is presented in Fig. 3.

\section{Vi. Performance Evaluation}

In this section, we present the simulation results for the delay upper bounds of cellular $\mathrm{V} 2 \mathrm{~V}, 6 \mathrm{G}$ communications, and hybrid $\mathrm{V} 2 \mathrm{~V}$ networks, respectively, and the proposed intelligent distance control strategy. In the traffic control simulation, we use the AirSim [32] simulator to conduct the

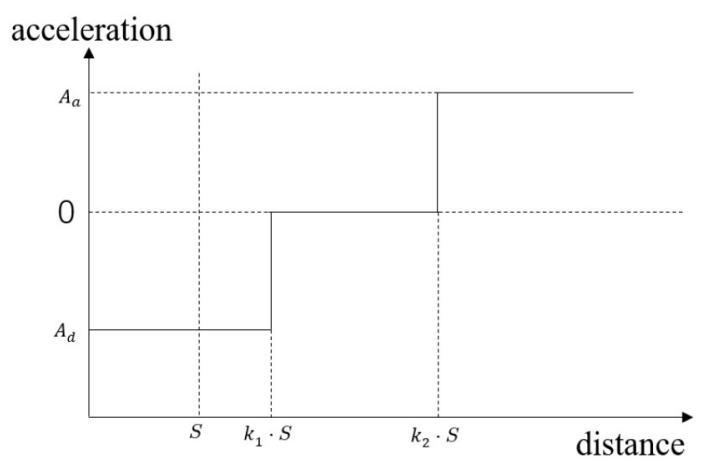

Fig. 2. Safe distance mapping.
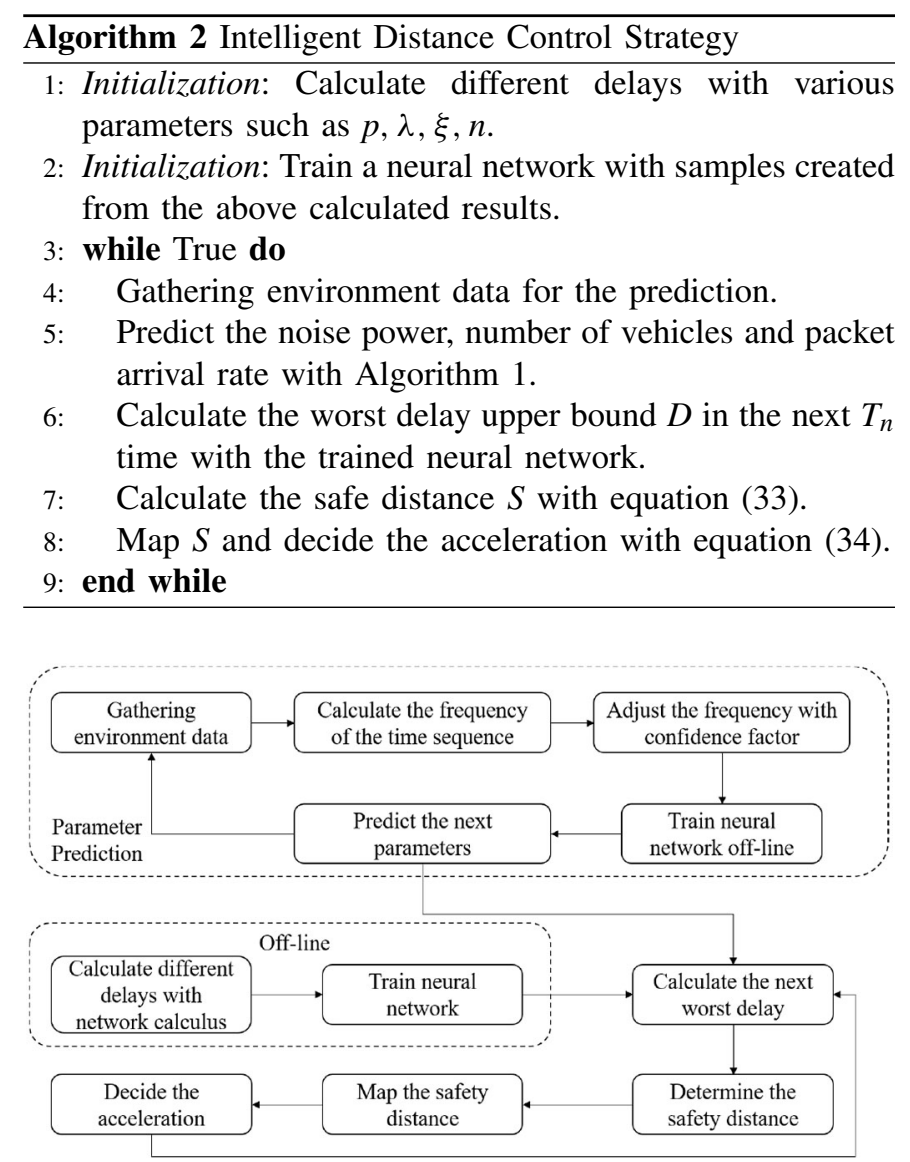

Fig. 3. Flowchart of the intelligent distance control strategy.

real-time evaluation of the proposed algorithms. As shown in Fig. 4, we implement an advanced network-level simulation platform, including AirSim for autonomous driving simulation, Mathematica for SNC calculation, machine learning, and communication simulation. PyZMQ is the Python bindings for the ZeroMQ library. The ZeroMQ library is required for the communication between the simulation processes of Mathematica and Python, which is an opensource universal messaging library to carry atomic messages across various transports by using sockets. In the autonomous driving simulation scenario shown in Fig. 5, a vehicular platoon with six vehicles is running on one lane. Some key parameters are presented in Table II. 
TABLE II

Key Parameter Settings in Simulation

\begin{tabular}{|c|c|c|c|}
\hline Parameter & Value & Parameter & Value \\
\hline SNR in (2) & $20 \mathrm{~dB}$ & $W$ & 4 \\
\hline$m$ & 3 & $M$ & 5 \\
\hline$t_{s}$ & 10 & $t_{C}$ & 5 \\
\hline$L$ & 100 & $l$ & $5 \mathrm{~m}$ \\
\hline
\end{tabular}

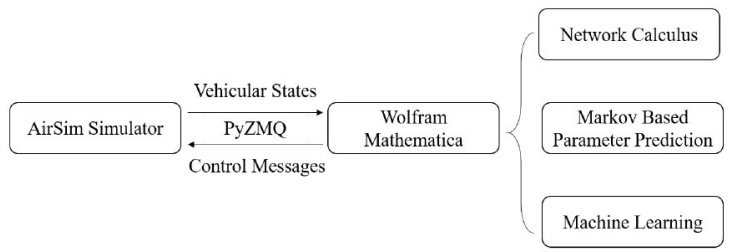

Fig. 4. Simulation framework.

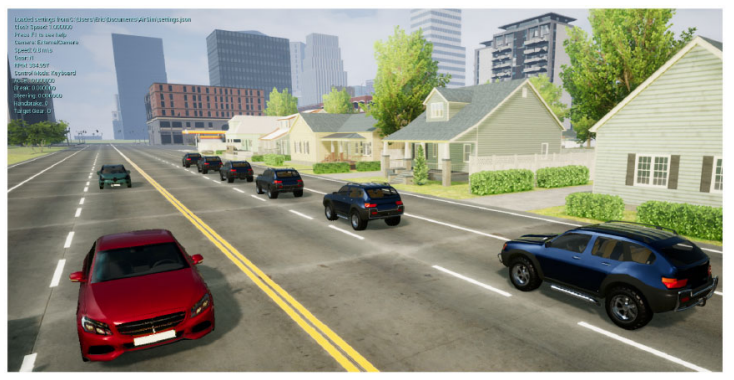

Fig. 5. Screen shot of the AirSim simulator.

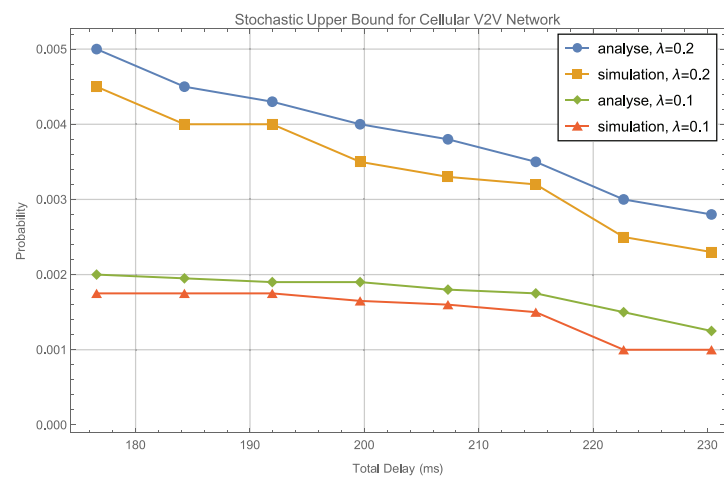

Fig. 6. Stochastic delay upper bound for pure cellular V2V network.

The computer that performs the proposed algorithms has a 2.9-GHz CPU with 8 cores, 16-GB RAM, a GTX 2060 GPU with 6-GB memory. For the SNC calculation, a complete calculation takes $28 \mathrm{~s}$ on average. For the deep neural network training, it takes $9.24 \mathrm{~s}$ to process 100 pieces of data. However, for the online prediction, the computer takes $0.5 \mathrm{~ms}$, which can satisfy the online processing requirement.

Fig. 6 shows the stochastic delay upper bound with pure cellular V2V communication. The number of vehicles is 10 in the simulation. The simulation delay is the probability of 10000 simulations. When the arrival rate is small $(\lambda=0.1)$, the tail of the delays' PDF keeps small and decreases in a gentle trend. However, when the arrival rate becomes high $(\lambda=0.2)$, the PDF decreases fast and becomes indistinguishable from the other PDF at the tail. Moreover, Fig. 6 also shows that

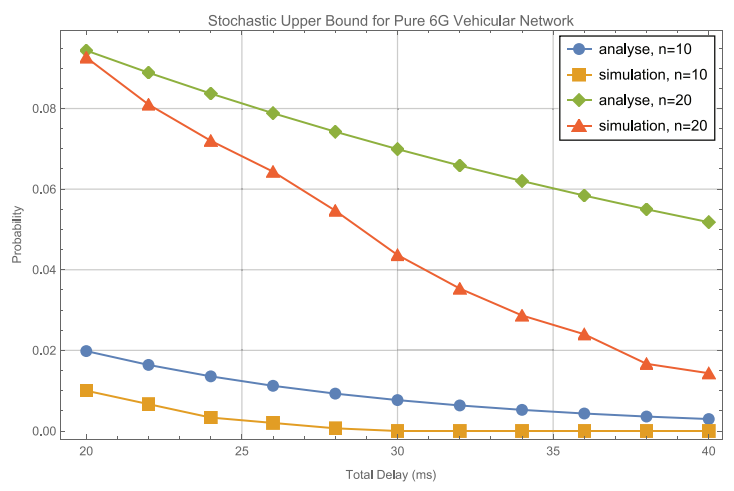

Fig. 7. Stochastic delay upper bound for pure $6 \mathrm{G}$ communication network.

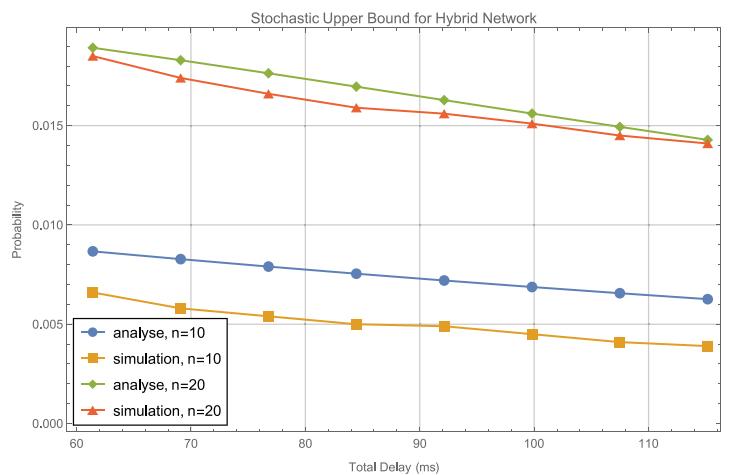

Fig. 8. Stochastic delay upper bound for hybrid V2V network

the delay upper bound with cellular V2V communication with competitive access protocols is very tight.

We illustrate the stochastic delay upper bound with mmWave communication in Fig. 7 as a typical representation of $6 \mathrm{G}$ vehicular communication technologies, where the packet arrival rate is 0.1 . The delay with mmWave is less than that with cellular V2V communication, due to the high speed of the mmWave links. However, the number of vehicles will affect the mmWave network dramatically. This is because multihop route increases the queue waiting time, which is the major contribution to the mmWave communication delay. As shown in Fig. 7, twice of the vehicle number increase the delay about four times.

In Fig. 8, the stochastic delay upper bound for hybrid $\mathrm{V} 2 \mathrm{~V}$ network is presented where packet arrival rate is 0.2 and the traffic is equally divided to be transmitted with mmWave and cellular V2V communications. Compare to that of cellular $\mathrm{V} 2 \mathrm{~V}$ communication, the tail probability with both communication technologies is smaller, which means a faster communication speed. This is because of the contribution of the mmWave communication. Moreover, compared with mmWave communication, the impact of the number of vehicles becomes lighter, which is caused by the one-hop communication pattern. As a result, the network with both communication technologies has better performance in most cases than the homogeneous networks.

Fig. 9 shows the average delay versus different packet arrival rate. When the packet arrival rate is small, the mmWave communication delivers the best performance. However, when the arrival rate increases, the performance with mmWave 


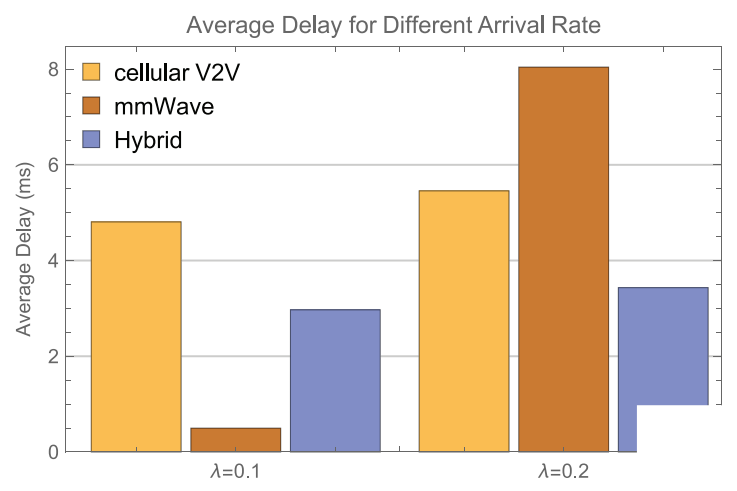

Fig. 9. Average delay for different arrival rates.

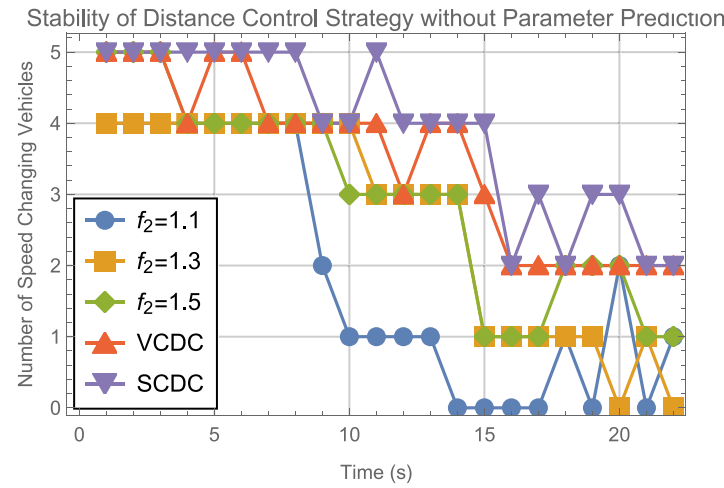

Fig. 10. Stability of distance control strategy.

communication drops dramatically. This is because that each vehicle generates packets in the same packet arrival rate, which makes the queue long in a high speed, which reduces the performance of the mmWave communication. The hybrid network can offload some of the traffic to the cellular V2V communication channel to keep the network in a normal state. Consequently, the hybrid network provides better performance guarantee than the vehicular networks with only mmWave communications.

We illustrate the stability of the intelligent distance control strategy in Fig. 10. We consider the algorithms with smoother vehicular speed changing and higher converge speed have higher stability. We compare the vehicular control algorithms only based on the sensor data in the traffic control fields, which is called string-constrained distributed control (SCDC) [26] and velocity-constrained distributed control (VCDC) [25], respectively. Compared to the algorithms without communication, the proposed algorithms converge much faster. At the beginning, there are six vehicles in the simulator and the distance between the adjacent vehicles is $10 \mathrm{~m}$ and the speed of the vehicular is $7.5 \mathrm{~m}$ per second. Because the communication condition becomes bad, the safe distance becomes longer and vehicles need to change their relative positions. The new safe distance is about $11.5 \mathrm{~m}$. Therefore, all of the vehicles beside the first vehicle need to decelerate. We count the number of vehicles that change their speeds at the different time slot. Lower $f_{2}$ means shorter safe distance mapping in (34). As a result, the vehicular platoon becomes stable faster if the $f_{2}$ is lower. However, all of the vehicular platoon become stable after $20 \mathrm{~s}$ with flat speed changing.

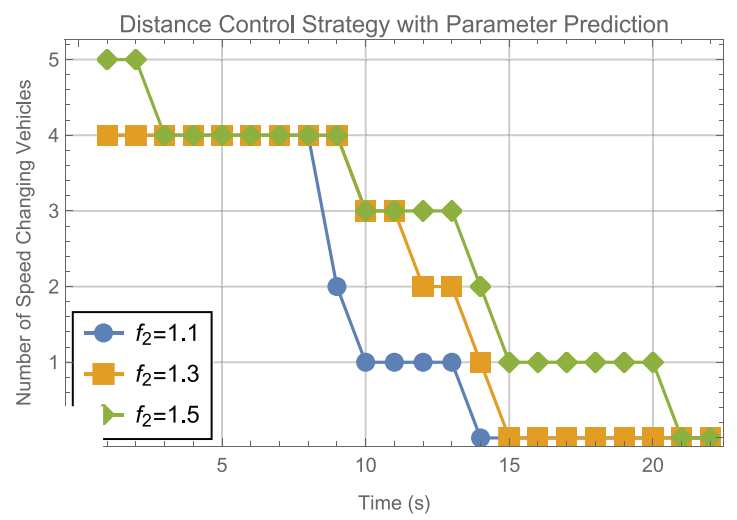

ance control strategy without parameter prediction.

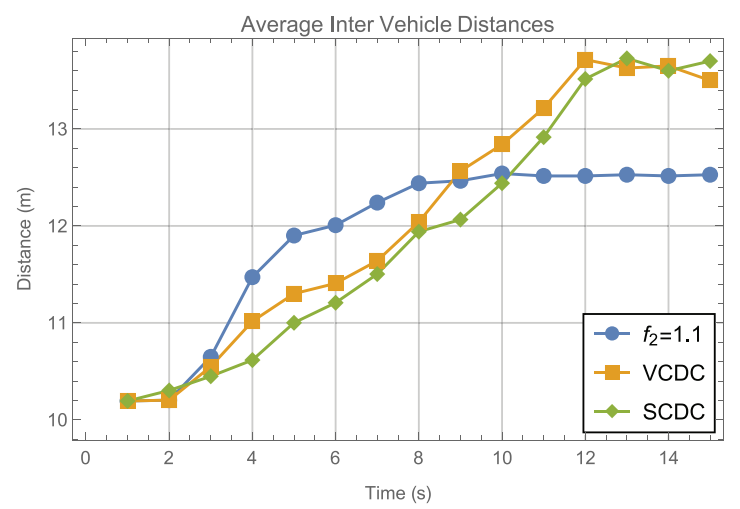

Fig. 12. Distances between vehicles in intelligent distance control strategy.

Fig. 11 illustrates the distance control strategy with parameter prediction in the same scenario with Fig. 10. Because all the vehicles decide their speeds only according to the current environment in the previous scenario, without parameter prediction, the vehicular platoon still fluctuates after becoming stable. With the parameter prediction algorithm, the number of speed changing vehicles becomes much less in the stable period.

Fig. 12 presents the average intervehicle distance of different algorithms. The simulation parameters are the same as the simulation in Fig. 10 where $f_{2}=1.1$. The distances become larger slowly and converge after $10 \mathrm{~s}$. After the platoon becomes stable, the distances fluctuate around the stable distance according to the delay upper bound. Compared with the other control algorithms, the intelligent distance control converges faster and becomes more stable at the end of simulation. In addition, the average intervehicle distance is much shorter than other algorithms, which means higher traffic efficiency than individual autonomous driving methods. Furthermore, according the traffic laws in China, the safety distance should be larger than $30 \mathrm{~m}$, which means the 6G supported cooperative autonomous driving with proposed distance control strategy has three times road capacity compare with manual driving methods, which is a dramatic improvement for road efficiency.

Furthermore, we conduct the simulation experiment for an urgent brake scenario. The first vehicle in the platoon starts to brake and send control messages through vehicular network. The speed of the last vehicle in the platoon is 


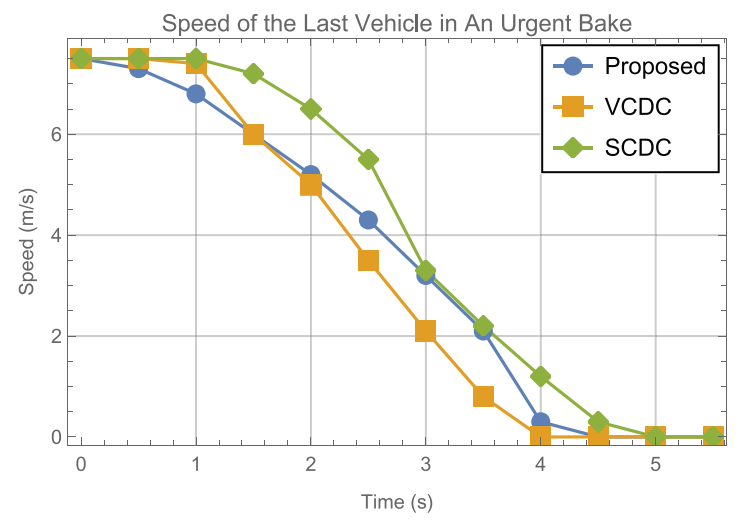

Fig. 13. Speed of the last vehicle in an urgent brake.

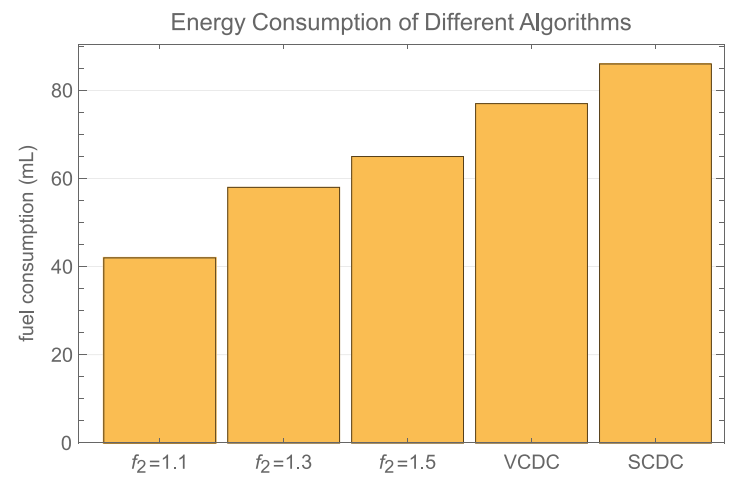

Fig. 14. Energy consumption of different algorithms.

recorded. From Fig. 13, we can find the last vehicle starts to brake in millisecond level, which is much faster than the reaction speed in the individual autonomous driving modes. This is because that the individual vehicles need to observe the behaviors of its preceding vehicle to decide its speed, which accumulate lots of reaction times along the lined platoon. Consequently, communication-based vehicle control can enhance safety because of the fast reaction for the accidents. Moreover, the smooth speed changing due to the proposed distance control algorithm can provide better driving experience compared to other control algorithms.

We compare the fuel consumption of different algorithms in Fig. 14. The simulation scenario is the same as Fig. 10. Since the intervehicle distances are relatively short in the starting phase of the simulation, the intervehicle distances need to be enlarged by decelerating the following vehicles. As a result, the algorithm with a large $f_{2}$ will need more speed reduction and result in more fuel consumption than that with a small $f_{2}$. Furthermore, the proposed algorithm with different parameters outperforms the SCDC and SCDC vehicle control methods. The proposed algorithm can save about $25 \%$ energy consumption compared to the other algorithms.

The above simulation results show that the network calculus can accurately model the delay upper bound. We can predict the delays precisely based on the deep understanding of the network states. Using the intelligent distance control algorithm, the platoon driving can become stable with smooth speed changing.

\section{CONCLUSION}

In this article, we studied the communication performance bounds for $6 \mathrm{G}$ vehicle networks with hybrid communication and channel access schemes. Based on the proposed SNC analytical model, we derive the performance of the cellular V2V communication networks and high-rate 6G communication networks. Then, the delay low bound of $\mathrm{V} 2 \mathrm{~V}$ communications is obtained by using the Stieltjes convolution. A deep learning-based approach was designed to predict the $\mathrm{V} 2 \mathrm{~V}$ communication performance bounds for real-time operation. Based on the performance guarantee analytical model, we proposed an intelligent intervehicle distance control strategy for cooperative driving. Moreover, a new parameter prediction algorithm was designed to reduce the number of speed changes for smooth driving. Extensive simulation experiments were conducted by an integrated simulation platform that combines communication module, analytical module, and AirSim driving module. Simulation results showed that the delay upper bound analysis and parameter prediction have sufficient accuracy for practical use. The proposed algorithms were very effective in maintaining intervehicle safe distance and stabilizing the cooperative driving platoons, which is helpful to improve driving safety, traffic, and energy efficiency. As the simulation results showed, the proposed algorithm can improve the road capacity about $10 \%$ than the existing individual autonomous driving-based safe distance control methods.

\section{REFERENCES}

[1] M. Green and J. Senders. Human Error in Road Accidents. Accessed: Nov. 2, 2020. [Online]. Available: https://www.visualexpert.com/ Resources/roadaccidents.html

[2] J. Zhou, D. Tian, Y. Wang, Z. Sheng, X. Duan, and V. C. M. Leung, "Reliability-optimal cooperative communication and computing in connected vehicle systems," IEEE Trans. Mobile Comput., vol. 19, no. 5, pp. 1216-1232, May 2020.

[3] J. He et al., "Cooperative connected autonomous vehicles (CAV): Research, applications and challenges," in Proc. IEEE 27th Int. Conf. Netw. Protocols (ICNP), 2019, pp. 1-6.

[4] K. Xiong, S. Leng, X. Chen, C. Huang, C. Yuen, and Y. L. Guan, "Communication and computing resource optimization for connected autonomous driving," IEEE Trans. Veh. Technol., vol. 69, no. 11, pp. 12652-12663, Nov. 2020.

[5] Y. Yang and K. Hua, "Emerging technologies for 5G-enabled vehicular networks," IEEE Access, vol. 7, pp. 181117-181141, 2019.

[6] G. Sun, Y. Zhang, H. Yu, X. Du, and M. Guizani, "Intersection fogbased distributed routing for $\mathrm{V} 2 \mathrm{~V}$ communication in urban vehicular ad hoc networks," IEEE Trans. Intell. Transp. Syst., vol. 21, no. 6, pp. 2409-2426, Jun. 2020.

[7] M. Zhang, R. Pan, and J. Shen, "A fast terahertz imaging: Method, simulation, and experiment," in Proc. 5th Glob. Symp. Millimeter Waves, 2012, pp. 431-434.

[8] E. C. Strinati et al., "6G: The next frontier: From holographic messaging to artificial intelligence using subterahertz and visible light communication," IEEE Veh. Technol. Mag., vol. 14, no. 3, pp. 42-50, Sep. 2019.

[9] P. Jiang, Z. Dong, Y. Wang, Y. Su, and Z. Li, "Empirical study of phantom traffic jams on urban expressway," in Proc. 9th Int. Conf. Intell. Control Inf. Process. (ICICIP), 2018, pp. 99-102.

[10] Z. Peng, S. Gao, Z. Li, B. Xiao, and Y. Qian, "Vehicle safety improvement through deep learning and mobile sensing," IEEE Netw., vol. 32, no. 4, pp. 28-33, Jul./Aug. 2018.

[11] R.-H. Huang, B.-J. Chang, Y.-L. Tsai, and Y.-H. Liang, "Mobile edge computing-based vehicular cloud of cooperative adaptive driving for platooning autonomous self driving," in Proc. IEEE 7th Int. Symp. Cloud Service Comput. (SC2), 2017, pp. 32-39. 
[12] K. Xiong, S. Leng, J. Hu, X. Chen, and K. Yang, "Smart network slicing for vehicular fog-RANs," IEEE Trans. Veh. Technol., vol. 68, no. 4, pp. 3075-3085, Apr. 2019.

[13] G. Qiao, S. Leng, K. Zhang, and Y. He, "Collaborative task offloading in vehicular edge multi-access networks," IEEE Commun. Mag., vol. 56, no. 8, pp. 48-54, Aug. 2018.

[14] X. Chen, H. Zhang, C. Wu, S. Mao, Y. Ji, and M. Bennis, "Optimized computation offloading performance in virtual edge computing systems via deep reinforcement learning," IEEE Internet Things J., vol. 6, no. 3 , pp. 4005-4018, Jun. 2019.

[15] X. Chen et al., "Age of information aware radio resource management in vehicular networks: A proactive deep reinforcement learning perspective," IEEE Trans. Wireless Commun., vol. 19, no. 4, pp. 2268-2281, Apr. 2020.

[16] S. Chen, K. Vu, S. Zhou, Z. Niu, M. Bennis, and M. Latva-Aho, "1 A deep reinforcement learning framework to combat dynamic blockage in mmWave V2X networks," in Proc. 2nd 6G Wireless Summit (6G SUMMIT), 2020, pp. 1-5.

[17] F. Tang, Y. Kawamoto, N. Kato, and J. Liu, "Future intelligent and secure vehicular network toward 6G: Machine-learning approaches," Proc. IEEE, vol. 108, no. 2, pp. 292-307, Feb. 2020.

[18] H. Zhou, W. Xu, J. Chen, and W. Wang, "Evolutionary V2X technologies toward the Internet of vehicles: Challenges and opportunities," Proc. IEEE, vol. 108, no. 2, pp. 308-323, Feb. 2020.

[19] Z. Yuan, Y. Ma, Y. Hu, and W. Li, "High-efficiency full-duplex V2Y communication," in Proc. 2nd 6G Wireless Summit (6G SUMMIT), 2020, pp. $1-5$.

[20] J. Xie and Y. Jiang, "A network calculus approach to delay evaluation of IEEE 802.11 DCF," in Proc. IEEE Local Comput. Netw. Conf., 2010, pp. 560-567.

[21] K. Katsaros, M. Dianati, R. Tafazolli, and X. Guo, "End-to-end delay bound analysis for location-based routing in hybrid vehicular networks," IEEE Trans. Veh. Technol., vol. 65, no. 9, pp. 7462-7475, Sep. 2016.

[22] G. Yang, M. Xiao, H. Al-Zubaidy, Y. Huang, and J. Gross, "Analysis of millimeter-wave multi-hop networks with full-duplex buffered relays," IEEE/ACM Trans. Netw., vol. 26, no. 1, pp. 576-590, Feb. 2018.

[23] K. Sakaguchi and R. Fukatsu, "Cooperative perception realized by millimeter-wave V2V for safe automated driving," in Proc. Asia Pac. Microw. Conf. (APMC), 2018, p. 180.

[24] P. Deng, Y. Zheng, J. Qiu, and L. Zhao, "Synchronous control of vehicle following behavior and distance under the safe and efficient steady-following state: Two case studies of high-speed train following control," IEEE Trans. Intell. Transp. Syst., vol. 19, no. 5, pp. 1445-1456, May 2018.

[25] J. C. Zegers, E. Semsar-Kazerooni, J. Ploeg, N. van de Wouw, and H. Nijmeijer, "Consensus control for vehicular platooning with velocity constraints," IEEE Trans. Control Syst. Technol., vol. 26, no. 5, pp. 1592-1605, Sep. 2018.

[26] S. Knorn, A. Donaire, J. C. Agüero, and R. H. Middleton, "Passivitybased control for multi-vehicle systems subject to string constraints," Automatica, vol. 50, no. 12, pp. 3224-3230, 2014. [Online]. Available: http://www.sciencedirect.com/science/article/pii/S0005109814004191

[27] H. Park, S. Park, T. Song, and S. Pack, "An incremental multicast grouping scheme for mmWave networks with directional antennas," IEEE Commun. Lett., vol. 17, no. 3, pp. 616-619, Mar. 2013.

[28] S. Rangan, T. S. Rappaport, and E. Erkip, "Millimeter-wave cellular wireless networks: Potentials and challenges," Proc. IEEE, vol. 102, no. 3, pp. 366-385, Mar. 2014.

[29] T. S. Rappaport, G. R. MacCartney, M. K. Samimi, and S. Sun, "Wideband millimeter-wave propagation measurements and channel models for future wireless communication system design," IEEE Trans. Commun., vol. 63, no. 9, pp. 3029-3056, Sep. 2015.

[30] J. Cho and Y. Jiang, "Basic theorems on the backoff process in 802.11," SIGMETRICS Perform. Eval. Rev., vol. 37, no. 2, p. 18-20, Oct. 2009. [Online]. Available: https://doi.org/10.1145/1639562.1639569

[31] Y. Jiang and Y. Liu, Stochastic Network Calculus. London, U.K.: Springer, 2009.

[32] S. Shah, D. Dey, C. Lovett, and A. Kapoor, "AirSim: High-fidelity visual and physical simulation for autonomous vehicles," in Proc. Field Service Robot. Conf., 2017, pp. 621-635. [Online]. Available: https://arxiv.org/abs/1705.05065

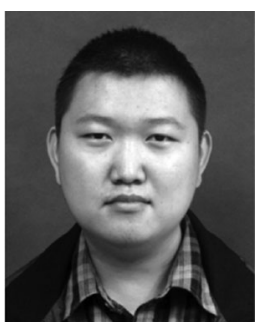

Xiaosha Chen received the B.Sc. degree in information and communication engineering from the University of Electronic Science and Technology of China, Chengdu, China, in 2016, where he is currently pursuing the Ph.D. degree.

His research interests include handling routing protocols, performance analysis, and congestion control in vehicular network.

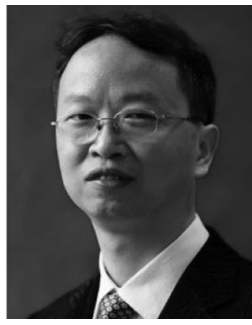

Supeng Leng (Member, IEEE) received the Ph.D. degree from Nanyang Technological University (NTU), Singapore in 2005 .

$\mathrm{He}$ is a Full Professor and a Vice Dean of the School of Information and Communication Engineering, University of Electronic Science and Technology of China, Chengdu, China, where he is also the Leader of the Research Group of Ubiquitous Wireless Networks. He has been working as a Research Fellow with the Network Technology Research Center, NTU. He published over 180 research papers in recent years. His research focuses on resource, spectrum, energy, routing, and networking in Internet of Things, vehicular networks, broadband wireless access networks, smart grid, and the next-generation mobile networks.

Prof. Leng serves as an Organizing Committee Chair and a TPC member for many international conferences, as well as a reviewer for over ten international research journals.

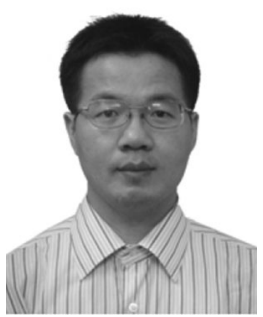

Jianhua He received the B.Sc. and M.Sc. degrees in electronic information engineering from the Huazhong University of Science and Technology (HUST), Wuhan, China, in 1995 and 1998, respectively, and the Ph.D. degree from Nanyang Technological University, Singapore, in 2002.

$\mathrm{He}$ is currently a Reader with University of Essex, Colchester, U.K. He has authored or coauthored more than 100 technical papers in major international journals and conferences. His main research interests include 5G networks, connected vehicles, autonomous driving, Internet of Things, AI for OCR, and wireless networks.

Dr. He is the Coordinator of EU Horizon2020 Project COSAFE on connected autonomous vehicles.

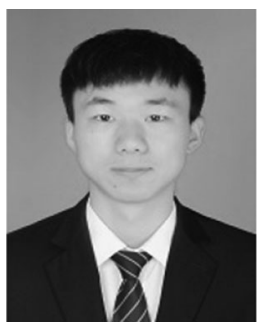

Longyu Zhou received the B.S. degree from Chongqing University of Posts and Telecommunications, Chongqing, China, in 2018. $\mathrm{He}$ is currently pursuing the Ph.D. degree with the School of Information and Communication Engineering, University of Electronic Science and Technology of China, Chengdu, China.

His current research include wireless sensor network, Internet of Things, edge intelligence, and resource management. 